$10,11,05$

\title{
Компьютерное моделирование фазовых переходов и критических свойств фрустрированной модели Гейзенберга на кубической решетке
}

\author{
(C) М.К. Рамазанов ${ }^{1,2}$, А.К. Муртазаев ${ }^{1,2}$ \\ ${ }^{1}$ Институт физики ДФИЦ РАН, \\ Махачкала, Россия \\ ${ }^{2}$ Дагестанский федеральный исследовательский центр РАН, \\ Махачкала, Россия \\ E-mail: sheikh77@mail.ru
}

Поступила в Редакцию 30 декабря 2019 г.

В окончательной редакции 30 декабря 2019 г.

Принята к публикации 10 января 2020 г.

\begin{abstract}
Методом Монте-Карло выполнены исследования фазовых переходов и критических свойств антиферромагнитной модели Гейзенберга на кубической решетке с учетом взаимодействий первых и вторых ближайших соседей. Рассмотрен диапазон значений величины взаимодействия вторых ближайших соседей $0.0 \leq r \geq 1.0$. Построена фазовая диаграмма зависимости критической температуры от величины взаимодействия вторых ближайших соседей. Показано, что в рассмотренном интервале значений $r$ наблюдается фазовый переход второго рода. Используя теорию конечно-размерного скейлинга, рассчитаны значения всех основных статических критических индексов. Показано, что класс универсальности критического поведения этой модели сохраняется в диапазоне значений $0.0 \geq r \geq 0.4$.
\end{abstract}

Ключевые слова: фрустрации, фазовые переходы, метод Монте-Карло, модель Гейзенберга.

DOI: $10.21883 /$ FTT.2020.06.49340.30M

\section{1. Введение}

В настоящее время продолжается активное исследование фазовых переходов (ФП) и критических свойств в спиновых системах с конкурирующим обменным взаимодействием. Конкуренция обменного взаимодействия может привести к возникновению в системе эффектов фрустрации. Наличие фрустраций в системе приводит к целому ряду изменений свойств фундаментального характера [1-4]. Спиновые системы с фрустрациями во многом проявляют свойства, отличные от соответствующих нефрустрированных систем. Это отличие выражается в богатом разнообразии фаз и ФП, сильным вырождением основного состояния и высокой чувствительности фрустрированных систем к различного рода возмущающим взаимодействиям [4,5].

В настоящее время получено достаточно большое количество интересных результатов для двумерной и трехмерной модели Изинга с конкурирующими обменными взаимодействиями на различных типах решеток [6-11]. ФП и критические свойства классической модели Гейзенберга с конкурирующими обменными взаимодействиями для трехмерного случая практически не исследованы.

В данной работе, нами на основе репличного алгоритма метода Монте-Карло (МК) проведено исследование ФП и критических свойств антиферромагнитной модели Гейзенберга на кубической решетке с учетом взаимодействий первых и вторых ближайших соседей в диапазоне $0.0 \geq r \geq 1.0$, где $r=J_{2} / J_{1}-$ величина взаимодействия вторых ближайших соседей $\left(J_{1}\right.$ и $J_{2}-$ константы обменного взаимодействия первых и вторых ближайших соседей, соответственно).

Интерес к исследуемой модели обусловлен тем что, при учете антиферромагнитных взаимодействий вторых ближайших соседей внутри слоев данная модель становится фрустрированной. В спиновых системах с фрустрациями многие физические свойства сильно зависят от величины взаимодействия вторых ближайших соседей. Кроме того, антиферромагнитная модель Гейзенберга на кубической решетке с учетом взаимодействия первых и вторых ближайших соседей до сих пор является малоизученной. Таким образом, исследование этой модели на основе современных методов позволит получить ответ на ряд вопросов, связанных с ФП и критическими свойствами фрустрированных спиновых систем.

\section{2. Модель и метод исследования}

Антиферромагнитная модель Гейзенберга с учетом взаимодействий первых и вторых ближайших соседей описывается следующим гамильтонианом:

$$
H=-J_{1} \sum_{\langle i, j\rangle}\left(\mathbf{S}_{j} \cdot \mathbf{S}_{j}\right)-J_{2} \sum_{\langle i, l\rangle}\left(\mathbf{S}_{j} \cdot \mathbf{S}_{l}\right)
$$

где $\mathbf{S}_{i}-$ трехкомпонентный единичный вектор $\mathbf{S}_{\mathbf{i}}=\left(\mathbf{S}_{i}^{x}, \mathbf{S}_{i}^{y}, \mathbf{S}_{i}^{z}\right)$. Решетка состоит из двумерных квадратных слоев, сложенных по ортогональной оси. Первый 
член в формуле (1) характеризует антиферромагнитное взаимодействие всех ближайших соседей, которое берется одинаковым как внутри слоев, так и между слоями $\left(J_{l}<0\right)$. Второй член характеризует антиферромагнитное взаимодействие вторых ближайших соседей, находящихся в том же слое $\left(J_{2}<0\right)$.

В настоящее время ФП и критические свойства фрустрированных спиновых систем на основе микроскопических гамильтонианов довольно успешно изучаются методами МК [12-18]. Методы МК позволяют исследовать физические свойства спиновых систем практически любой сложности. На их основе, на сегодняшний день, изучены целые классы спиновых систем и рассчитаны критические индексы широкого спектра моделей. В данном исследовании был использован репличный обменный алгоритм метода МК [19], который является наиболее мощным и эффективным инструментом для исследования фрустрированных спиновых систем.

\section{3. Результаты моделирования}

Расчеты проводились для систем с периодическими граничными условиями и с линейными размерами $L \times L \times L=N, L=24-60$. Для вывода системы в состояние термодинамического равновесия отсекался участок длиной $\tau_{0}=4 \cdot 10^{5} \mathrm{MK}$ шагов/спин, что в несколько раз больше длины неравновесного участка. Усреднение термодинамических величин проводилось вдоль марковской цепи длиной $\tau==500 \tau_{0}$ МК шагов/спин.

Для наблюдения за температурным ходом теплоемкости $C$ и восприимчивости $c h i$ использовались выражения [20]:

$$
\begin{gathered}
C=\left(N K^{2}\right)\left(\left\langle U^{2}\right\rangle-\langle\rangle^{2}\right), \\
\chi= \begin{cases}(N K)\left(\left\langle m^{2}\right\rangle-\langle|m|\rangle^{2}\right), & T<T_{N} \\
(N K)\left\langle m^{2}\right\rangle, & T \geq T_{N}\end{cases}
\end{gathered}
$$

где $K=\left|J_{1}\right| / k_{B} T, N-$ число частиц, $T_{N}$ - критическая температура, $U-$ внутренняя энергия, $m$ - параметр порядка ( $U$ и $m$ являются нормированными величинами).

Расчет параметра порядка системы m был выполнен на основе выражения вида:

$$
\begin{gathered}
m_{i}=\frac{4}{N} \sum_{i \in \lambda}(-1)^{z} \mathbf{S}, \quad \text { где } \lambda=1,2,3,4, \\
m^{a}=\left[m_{1}+m_{2}-\left(M_{3}+m_{4}\right)\right] / 4, \\
m^{b}=\left[m_{1}+m_{4}-\left(m_{2}+m_{3}\right)\right] / 4, \\
m=\sqrt{\left(m^{a}\right)^{2}+\left(m^{b}\right)^{2}}
\end{gathered}
$$

где $m_{1}, m_{2}, m_{3}, m_{4}$ - параметр порядка по подрешеткам, $z$ - номер слоя решетки.

На рис. 1 и 2 представлены температурные зависимости параметра порядка при $L=30$ для различных значений $r$ (здесь и далее статистическая погрешность не превышает размеров символ, использованных для

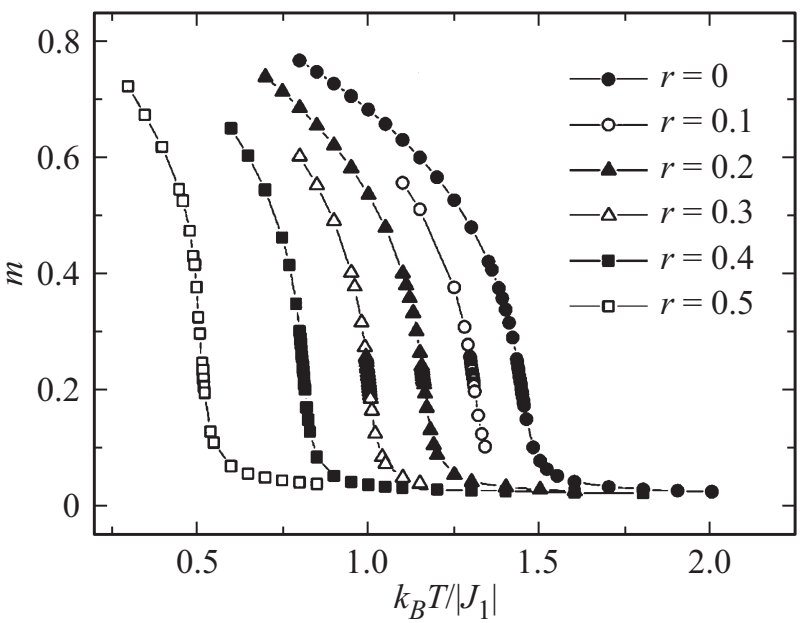

Рис. 1. Зависимость параметра порядка $m$ от температуры $k_{B} T /\left|J_{1}\right|$ для значений $r$ в интервале $0 \leq r \leq 0.5$.

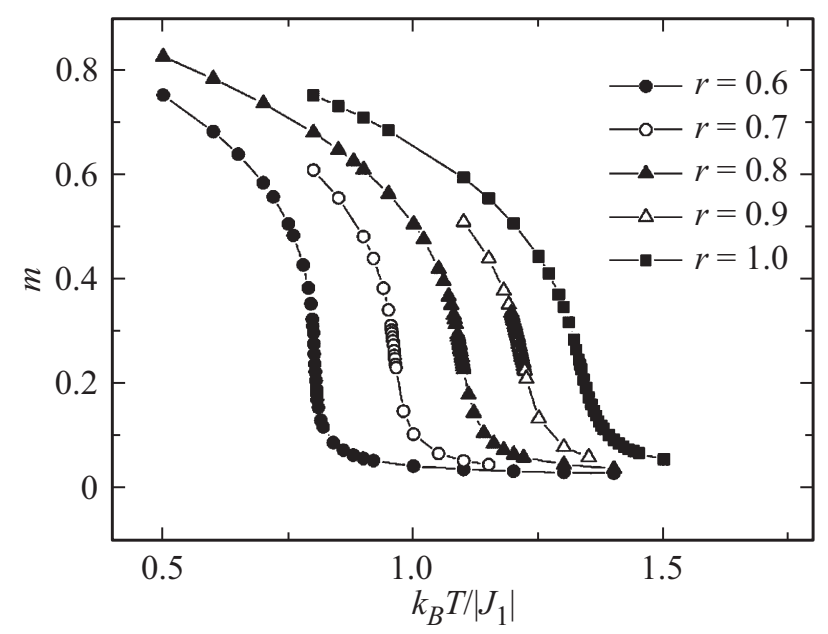

Рис. 2. Зависимость параметра порядка $m$ от температуры $k_{B} T /\left|J_{1}\right|$ для значений $r$ в интервале $0.6 \leq r \leq 1.0$.

построения зависимостей). Отметим, что с увеличением значения $r$ в интервале $0.0 \leq r \leq 0.5$ спад параметра порядка сдвигается в сторону более низких температур. В интервале $0.6 \leq r \leq 1.0$, мы наблюдаем противоположную картину. С увеличением $r$ от 0.6 до 1.0 спад параметра порядка смещается в сторону более высоких температур.

На рис. 3 и 4 представлены температурные зависимости восприимчивости, полученные при $L=30$ для различных значений $r$. Отметим, что увеличение значения $r$ в интервале $0.0 \leq r \leq 0.5$ сопровождается сдвигом максимумов в сторону более низких температур, одновременно с этим наблюдается рост абсолютных значений максимумов восприимчивости. Рост абсолютных значений максимумов происходит за счет конкуренции первых и вторых ближайших соседей. В случае, когда $0.6 \leq r \leq 1.0$, наблюдаем противоположную картину. С увеличением $r$ от 0.6 до 1.0 наблюдается спад 


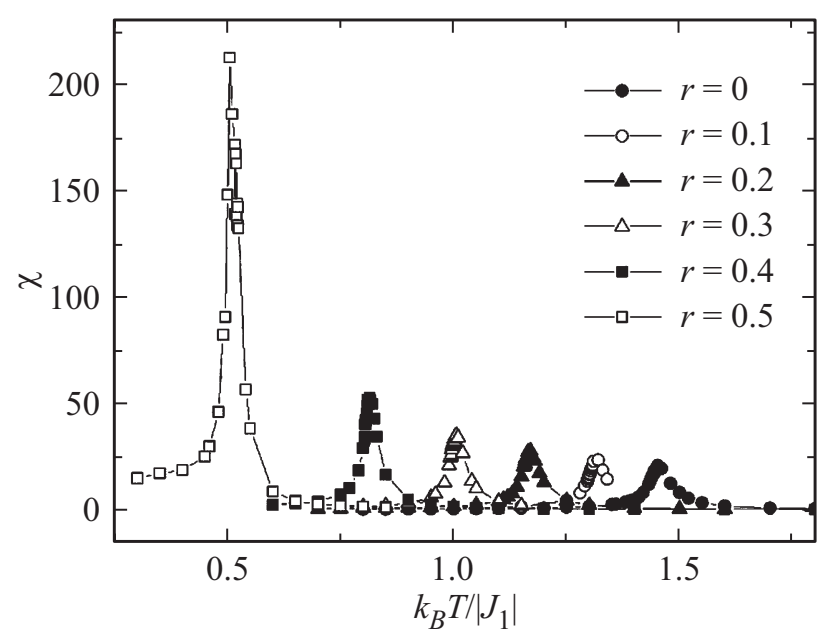

Рис. 3. Зависимость восприимчивости $\chi$ от температуры $k_{B} T /\left|J_{1}\right|$ для значений $r$ в интервале $0 \leq r \leq 0.5$.

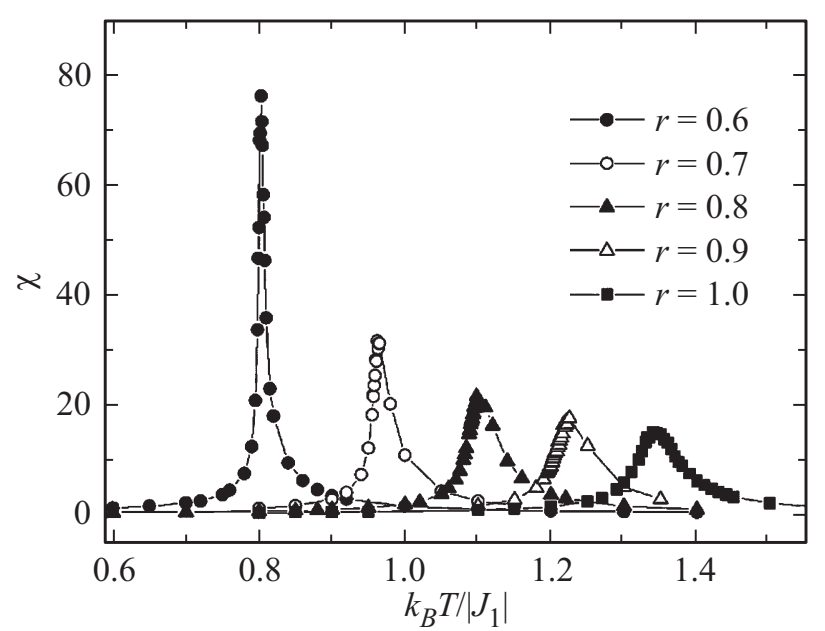

Рис. 4. Зависимость восприимчивости $\chi$ от температуры $k_{B} T /\left|J_{1}\right|$ для значений $r$ в интервале $0.6 \leq r \leq 1.0$.

абсолютных значений максимумов восприимчивости и максимумы смещаются в сторону более высоких температур.

Увеличение взаимодействия вторых ближайших coceдей в этом интервале, приводит к увеличению энергии взаимодействия по модулю, что укрепляет жесткость системы и соответственно повышается температура фазового перехода.

Для определения критической температуры $T_{N}$, нами использовался метод кумулянтов Биндера $U_{L}$ четвертого порядка [21]:

$$
U_{L}=1-\frac{\left\langle m^{4}\right\rangle}{3\left\langle m^{2}\right\rangle_{L}^{2}},
$$

Согласно теории конечноразмерного скейлинга точка пересечения всех кривых $U_{L}(T)$ является критической точкой [21]. Выражение (8) позволяет определить критическую температуру $T_{N}$ с большой точностью.
На рис. 5 представлены характерные зависимости $U_{L}$ от температуры при $r=0.7$ для разных значений $L$. Рисунок демонстрирует точность определения критической температуры. Видно, что в критической области наблюдается четко выраженная точка пересечения $\left(T_{N}=0.963\right.$; здесь и далее температура дана в единицах $\left.\left|J_{1}\right| / k_{B}\right)$. Аналогичным образом были определены критические температуры и для остальных значений $r$.

На рис. 6 приведена фазовая диаграмма зависимости критической температуры от величины взаимодействия вторых ближайших соседей.

На диаграмме видно, что вблизи значения $r=0.5$ пересекаются три различные фазы: антиферромагнитная $I$, парамагнитная - II и суперантиферромагнитная III. Как было показано в работе [22], для всех рассматриваемых значений $\mathrm{r}$ наблюдаются ФП второго рода.

Для расчета статических критических индексов теплоемкости $\alpha$, параметра порядка $\beta$, восприимчивости $\gamma$ и радиуса корреляции $v$ применялись соотношения теории конечно-размерного скейлинга. Из теории конечно-

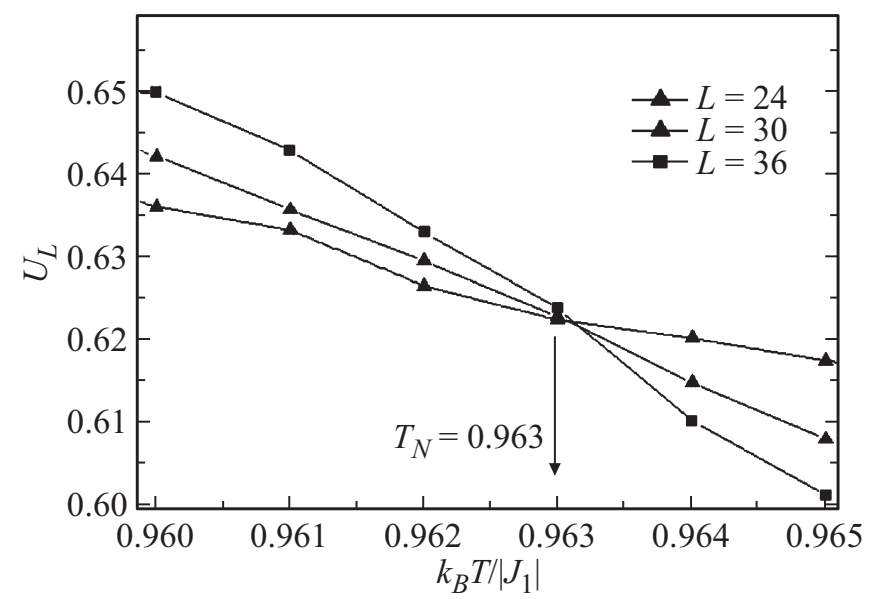

Рис. 5. Зависимости кумулянта Биндера $U_{L}$ от температуры $k_{B} T /\left|J_{1}\right|$ для $r=0.7$ при различных $L$.

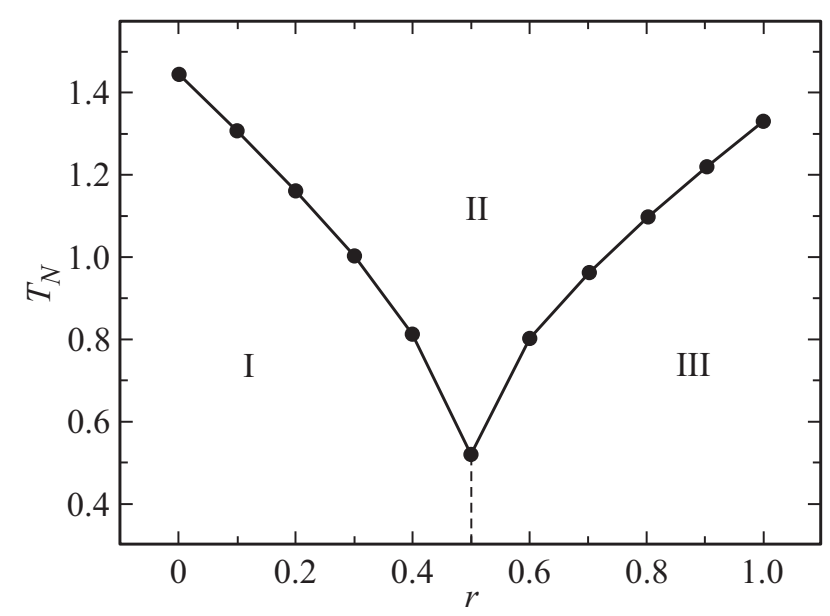

Рис. 6. Фазовая диаграмма зависимости критической температуры от величины взаимодействия вторых ближайших соседей. 
Значения коиьических параметров для антиферромагнитной модели Гейзенберга на кубической решетке

\begin{tabular}{c|c|c|c|c|c|c|c}
\hline$r$ & $k_{B} T_{N}\left|J_{1}\right|$ & $v$ & $\alpha$ & $\beta$ & $\gamma$ & $\eta$ & $\alpha+2 \beta+\gamma=2$ \\
\hline $\begin{array}{c}\text { Нефрустрированная } \\
\text { модель }\end{array}$ & $1.443(1)$ & $0.7112(5)$ & $-0.01336(15)$ & $0.3689(3)$ & $1.3960(9)$ & $0.0375(5)$ & \\
Гейзенберга [22] & & & & & & \\
\hline 0.0 & $1.443(1)$ & $0.712(5)$ & $-0.134(5)$ & $0.366(5)$ & $1.393(5)$ & $0.036(5)$ & 2 \\
0.1 & $0.307(1)$ & $0.710(5)$ & $-0.132(5)$ & $0.364(5)$ & $1.390(5)$ & $0.032(5)$ & 1.98 \\
0.2 & $1.160(1)$ & $0.711(5)$ & $-0.133(5)$ & $0.362(5)$ & $0.392(5)$ & $1.034(5)$ & 1.98 \\
0.3 & $1.000(1)$ & $0.713(5)$ & $-0.136(5)$ & $0.365(5)$ & $1.391(5)$ & $0.033(5)$ & 1.99 \\
0.4 & $0.811(1)$ & $0.712(5)$ & $-0.133(5)$ & $0.363(5)$ & $1.393(5)$ & $0.035(5)$ & 1.99 \\
0.6 & $0.802(1)$ & $0.650(5)$ & $0.161(5)$ & 0.295() 5 & $1.259(5)$ & $0.06(2)$ & 2.01 \\
0.7 & $0.963(1)$ & $0.648(5)$ & $0.163(5)$ & $0.293(5)$ & $1.256(5)$ & $0.05(2)$ & 2 \\
0.9 & $1.096(1)$ & $1.647(5)$ & $0.162(5)$ & $0.291(5)$ & $1.258(5)$ & $0.05(2)$ & 2 \\
1.0 & $1.219(1)$ & $0.651(5)$ & $0.164(5)$ & $0.292(5)$ & $1.257(5)$ & $0.07(2)$ & 2.01 \\
\end{tabular}

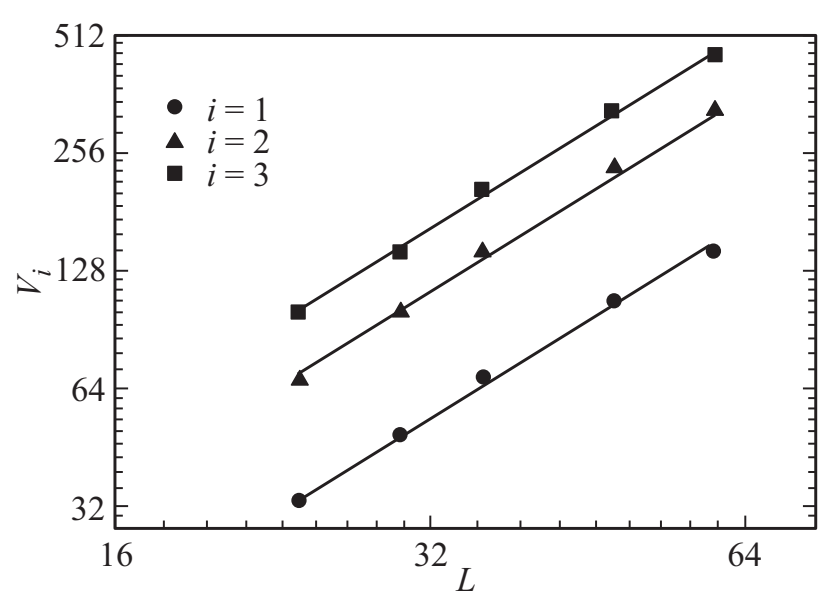

Рис. 7. Зависимость параметра $V_{i}$ от линейных размеров системы $L$ при $T=T_{N}$ для $r=0.7$.

размерного скейлинга следует, что в системе с размерами $L \times L \times L$ при $k_{B} T /\left|J_{1}\right|=k_{B} T_{N} /\left|J_{1}\right|$ и достаточно больших $L$ выполняются следующие выражения [23-25]:

$$
\begin{aligned}
m & \sim L^{-\beta / v}, \\
\chi & \sim L^{\gamma / v}, \\
V_{i} & \sim L^{1 / v} g_{V_{i}},
\end{aligned}
$$

где $g_{V_{i}}$ - постоянная, а в качестве $V_{i}$ могут выступать

$$
V_{i}=\frac{\left\langle m^{i} E\right\rangle}{\left\langle m^{i}\right\rangle}-\langle E\rangle, \quad(i=1,2,3) .
$$

Эти выражения были нами использованы для определения значений критических индексов $\beta, \gamma$ и $\nu$.

Для аппроксимации температурной зависимости теплоемкости от $L$ на практике, как правило, используется выражение [6]:

$$
C_{\max }(L)=A_{1}-A_{2} L^{\alpha / v},
$$

где $A_{1}$ и $A_{2}$ - некоторые коэффициенты.
На рис. 7 в двойном логарифмическом масштабе представлены характерные зависимости параметров $V_{i}$ при $i=1,2,3$ от линейных размеров решетки $L$ для $r=0.7$. Как видно из рисунка все точки на графиках в пределах погрешности хорошо ложатся на прямую. Зависимости на рисунке, проведенные в соответствии с методом наименьших квадратов, параллельны друг другу. Угол наклона прямой определяет значение $1 / v$. Вычисленное таким образом значение $v$ использовалось для определения критических индексов теплоемкости $\alpha$, параметра порядка $\beta$ и восприимчивости $\gamma$.

На рис. 8 и 9 в двойном логарифмическом масштабе представлены характерные зависимости магнитного параметра порядка $m$ и восприимчивости $\chi$ от линейных размеров решетки $L$ для $r=0.7$. Все точки в пределах погрешности ложатся на прямые. Углы наклона этих прямых определяют значения $\beta / v$ и $\gamma / v$. По этой схеме было определено значение и для теплоемкости $\alpha / v$. Имея данные по $v$, вычислялись статические критические индексы $\alpha, \beta$ и $\gamma$.

Эта процедура использовалась для расчета критических индексов для всех рассмотренных значений $r$. Все значения статических критических индексов, полученные таким образом, представлены в таблице. Процедура, использованная нами для определения индекса Фишера $\eta$ более подробно изложена в работе [26]. Данные, полученные нами для индекса Фишера $\eta$ также представлены в таблице.

Отметим, что для значения $r=0.5$ рассчитать критические индексы с допустимой погрешностью не удалось. Предполагаем, что это связано с тем, что в этой точке сосуществуют три различные фазы.

Как видно в таблице, критическая температура $k_{B} T_{N} /\left|J_{1}\right|$ уменьшается с увеличением величины взаимодействия вторых ближайших соседей вплоть до значения $r=0.6$. При дальнейшем увеличении $r$ критическая температура начинает расти. Все значения критических индексов, рассчитанные нами в интервале $0.0 \leq r \leq 0.4$, в пределах погрешности совпадают со значениями кри- 


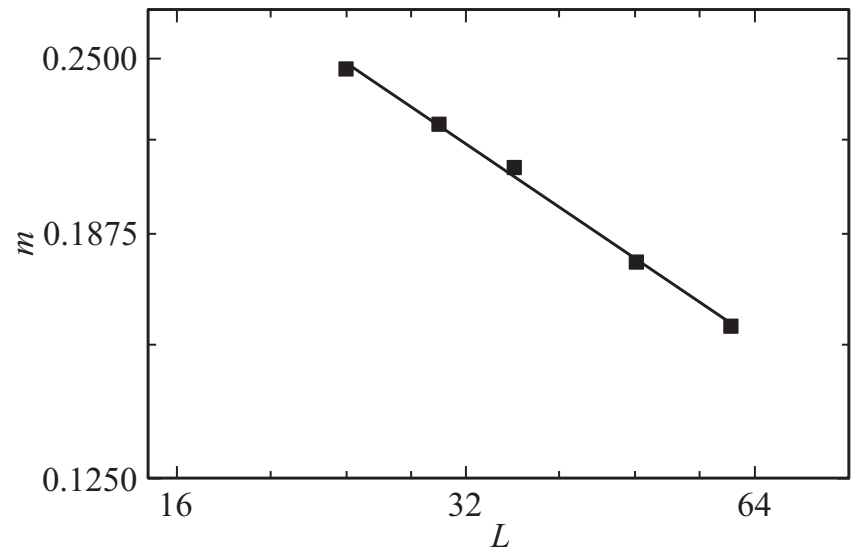

Рис. 8. Зависимость параметра порядка $m$ от линейных размеров системы $L$ при $T=T_{N}$ для $r=0.7$.

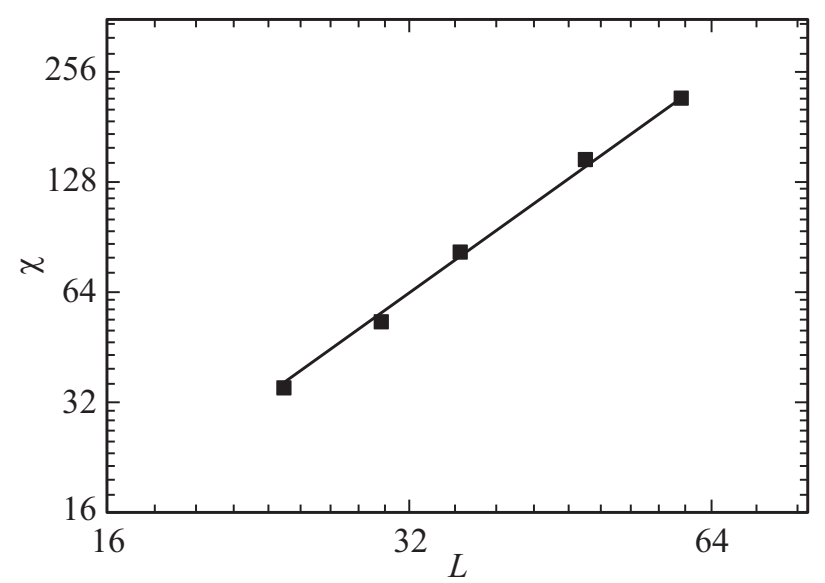

Рис. 9. Зависимость восприимчивости $\chi$ от линейных размеров системы $L$ при $T=T_{N}$ для $r=0.7$.

тических индексов трехмерной нефрустрированной модели Гейзенберга [27]. Это свидетельствует о принадлежности данной модели в интервале $0.0 \leq r \leq 0.4$ к тому же классу универсальности критического поведения, что и нефрустрированная модель Гейзенберга. Значения критических индексов, рассчитанные нами в интервале $0.6 \leq r \leq 1.0$ сильно отличаются от данных, полученных в интервале $0.0 \leq r \leq 0.4$. Можно предположить, что учет взаимодействий вторых ближайших соседей внутри слоев решетки для антиферромагнитной модели Гейзенберга на кубической решетке приводит к смене класса универсальности критического поведения.

\section{4. Заключение}

Исследование фазовых переходов и критического поведения антиферромагнитной модели Гейзенберга на кубической решетке с учетом взаимодействия первых и вторых ближайших соседей внутри слоев решетки выполнено с использованием высокоэффективного репличного алгоритма метода Монте-Карло. Построена фа- зовая диаграмма зависимости критической температуры от величины взаимодействия вторых ближайших соседей. Определены значения критических температур и рассчитаны значения всех основных статических критических индексов в интервале $0.0 \leq r \leq 1.0$. Установлены закономерности изменения критических параметров в рассмотренном интервале $r$. Обнаружено, что в интервале $0.0 \leq r \leq 0.4$ система проявляет универсальное критическое поведение. Показано, что в рассматриваемой модели в интервале $0.6 \leq r \leq 1.0$ наблюдается другое критическое поведение.

\section{Финансирование работы}

Исследование выполнено при финансовой поддержке Российского фонда фундаментальных исследований в рамках научных проектов № 19-02-00153-а и 18-3220098-мол-а-вед.

\section{Конфликт интересов}

Авторы заявляют, что у них нет конфликта интересов.

\section{Список литературы}

[1] Вик.С. Доценко. УФН 165, 481 (1995).

[2] С.Е. Коршунов. УФН 176, 233 (2006).

[3] С.В. Малеев. УФН 172, 617 (2002).

[4] M.K. Ramazanov, A.K. Murtazaev, M.A. Magomedov. Solid State Comm. 233, 35 (2016).

[5] D.P. Landau, K. Binder. Monte Carlo Simulations in Statistical Physics. Cambridge University Press, Cambridge (2000). P. 384.

[6] А.К. Муртазаев, М.К. Рамазанов, М.К. Бадиев. ФНТ 37, 1258 (2011).

[7] A. Kalz, A. Honecker. Phys. Rev. B 86, 134410 (2012).

[8] S. Jin, A. Sen, A.W. Sandvik. Phys. Rev. Lett. 108, 045702 (2012).

[9] S. Jin, A. Sen, W. Guo, A.W. Sandvik. Phys. Rev. B 87, 144406 (2013).

[10] М.К. Рамазанов, А.К. Муртазаев. Письма в ЖЭТФ 101, 793 (2015).

[11] М.К. Рамазанов, А.К. Муртазаев. Письма в ЖЭТФ 103, 522 (2016).

[12] A.K. Murtazaev, M.K. Ramazanov, D.R. Kurbanova, M.A. Magomedov, K.Sh. Murtazaev. Mater. Lett. 236, 669 (2019).

[13] A.K. Murtazaev, M.K. Ramazanov, M.K. Badiev. Physica A 507, 210 (2018).

[14] M.K. Ramazanov, A.K. Murtazaev, M.A. Magomedov. Physica A 521, 543 (2019).

[15] А.К. Муртазаев, М.К. Рамазанов, М.К. Мазагаева, М.А. Магомедов. ЖЭТФ 156, 502 (2019).

[16] А.К. Муртазаев, М.К. Рамазанов, Д.Р. Курбанова, М.К. Бадиев. ФТТ 60, 1162 (2018).

[17] А.К. Муртазаев, М.К. Рамазанов, Д.Р. Курбанова, М.А. Магомедов, М.К. Бадиев, М.К. Мазагаева. ФТТ 61, 1170 (2019). 
[18] А.К. Муртазаев, М.К. Рамазанов, М.К. Бадиев. ФТТ 61, 1898 (2019).

[19] A. Mitsutake, Y. Sugita, Y. Okamoto. Biopolymers (Peptide Science) 60, 96 (2001).

[20] K. Binder, J.-Sh. Wang. J. Status. Phys. 55, 87 (1989).

[21] K. Binder, D.W. Heermann. Monte Carlo Simulation in Statistical Physics. Springer_Verlag, Berlin (1988); Nauka, M. (1995). P. 214.

[22] М.К. Рамазанов, А.К. Муртазаев. Письма в ЖЭТФ 109, 610 (2019).

[23] A.E. Ferdinand, M.E. Fisher. Phys. Rev. 185, 832 (1969).

[24] M.E. Fisher, M.N. Barber. Phys. Rev. Lett. 28, 1516 (1972).

[25] P. Peczak, A.M. Ferrenberg, D.P. Landau. Phys. Rev. B 43, 6087 (1991).

[26] А.К. Муртазаев, М.К. Рамазанов. ФТТ 59, 1797 (2017).

[27] M. Campostrini, M. Hasenbusch, A. Pelissetto. Phys. Rev. B 65, 144520 (2002).

Редактор Т.Н. Василевская 